\title{
Sistema de Monitoreo y Control Automatizado de Nivel para Contenedores de Líquidos no Corrosivos, de Uso Doméstico y Comercial
}

\section{Automated Level Monitoring and Control System for Containers of Non-Corrosive Liquids, for Domestic and Commercial Use}

BÁEZ-IBARRA, Alejandro ${ }^{1} \dagger^{*}$, ENRÍQUEZ-NICOLÁS, Mario ${ }^{2}$, LUNA-SALINAS, Francisco Antonio $^{2}$ y ROJAS-SANTOS, Guadalupe ${ }^{1}$

${ }^{1}$ Instituto Tecnológico de Pochutla

${ }^{2}$ Instituto Tecnológico de Salina Cruz.

ID 1 ${ }^{\text {er }}$ Autor: Alejandro, Báez-Ibarra / ORC ID: 0000-0002-1935-2482, CVU CONACYT ID: 537153

ID $1^{\text {er }}$ Coautor: Mario, Enríquez-Nicolás /

ID $2^{\text {do }}$ Coautor: Francisco Antonio, Luna-Salinas /

ID $3^{\text {er }}$ Coautor: Guadalupe, Rojas-Santos / ORC ID: 0000-0002-6743-065X y CVU CONACYT ID: 999419

DOI: $10.35429 / J T D .2019 .11 .3 .1 .8$

Recibido: 20 de Julio, 2018; Aceptado 10 de Septiembre, 2018

\begin{abstract}
Resumen
La automatización de procesos a día de hoy, es una de las necesidades presentes en la sociedad como consecuencia de lo ineficiente que resulta ser la realización de ciertas tareas sin mecanismos que las optimicen, ya sea por cuestiones de tiempo, eficiencia, y en general por todo el avance tecnológico que ya se tiene, estos mecanismos han quedado obsoletos. El presente documento, muestra una respuesta a esta situación, específicamente enfocada a procesos de gestión de líquidos, la implementación de un Sistema de Monitoreo y Control Automatizado de Nivel para Contenedores no Corrosivos de uso doméstico como comercial. La adecuada selección de los componentes que integran este sistema permitirá su fácil accesibilidad, con respecto al costo que ello implica, su estructura estará diseñada de tal forma que reduzca el mínimo el tiempo de ejecución, que integre más de un control, y que su interfaz sea lo más amigable posible con el operador. Esto permite, que sea un sistema fácil de usar, mejorando en gran medida el tiempo de respuesta en su realización, así también, permitirá tener un mayor control en la capacidad del nivel de los contenedores reduciendo de esta forma el gasto de líquidos que se puede suscitar por algún descuido.
\end{abstract}

Monitoreo, Contenedores, Líquidos

\begin{abstract}
The automation of processes today is one of the present needs in society as a result of the inefficiency that results from carrying out certain tasks without mechanisms that optimize them, whether due to time, efficiency, and in general All the technological progress that we already have, these mechanisms have become obsolete. This document shows a response to this situation, specifically focused on liquid management processes, the implementation of a Level Automated Monitoring and Control System for non-corrosive containers for domestic and commercial use. The adequate selection of the components that make up this system will allow its easy accessibility, with respect to the cost that this implies, its structure will be designed in such a way that it minimizes the execution time, that integrates more than one control, and that its interface Be as friendly as possible with the operator. This allows it to be an easy-to-use system, greatly improving the response time in its realization, as well as it will allow to have a greater control in the capacity of the level of the containers reducing in this way the expenditure of liquids that can be arouse by some carelessness.
\end{abstract}

Monitoring, Containers, Liquids

Citación: BÁEZ-IBARRA, Alejandro, ENRÍQUEZ-NICOLÁS, Mario, LUNA-SALINAS, Francisco Antonio y ROJASSANTOS, Guadalupe. Sistema de Monitoreo y Control Automatizado de Nivel para Contenedores de Líquidos no Corrosivos, de Uso Doméstico y Comercial. Revista del Desarrollo Tecnológico. 2019 3-11: 1-8

\footnotetext{
* Correspondencia del Autor (Correo electrónico: abaezi@itpochutla.edu.mx)

$\dagger$ Investigador contribuyendo como primer autor.
} 


\section{Introducción}

Tener un sistema que automatice un proceso en la actualidad es una enorme diferencia en contraste aquellos que no lo tienen, no solo por el hecho de reducir el trabajo, sino más aún porque hace eficiente la ejecución, e incrementa el tiempo para la realización de otros procesos que así lo requieran. En el Instituto Tecnológico de Pochutla, se presenta la situación de la falta de un sistema que monitoree el llenado de los tanques utilizados para abastecer a todo el alumnado, y personal administrativo, y que cuente con características específicas, brindando la elección de ser tanto de uso doméstico como comercial, además de ampliar su adquisición por su bajo costo, al estar formado por componentes exactos que cumplan con su funcionamiento, y en suma sea fácil de usar. En las siguientes secciones se presenta, la metodología para la creación de un sistema y sus partes, explicando detalladamente el proceso de desarrollo, los resultados y las conclusiones obtenidas al finalizar su creación.

\section{Método y metodología}

Con el propósito de comprender a profundidad y precisión el objeto de estudio en cuestión, ello implica su funcionamiento, fue necesario conocer detalladamente cada una de las partes que lo componen, y que resultan ser imprescindibles para su correcta ejecución, de este modo, al estudiarlas de forma particular, fue posible determinar las relaciones que existen entre ellas, y la manera en cómo estas afectan la operatividad total del sistema, conociendo así la naturaleza misma del fenómeno. Esto permite que, al comenzar partiendo de lo concreto a lo abstracto, sea posible la incorporación de nuevos conocimientos, con lo cual se obtienen mejores resultados a medida en que todas sus partes son examinadas.

Por lo anterior, y teniendo en cuenta que el objetivo principal del desarrollo del tema de investigación, fue la creación de un sistema de monitorio y control automatizado de nivel para contenedores de líquidos no corrosivos de uso tanto doméstico como comercial, y aunado a que a su vez tuviese las particularidades de ser un sistema eficiente, eficaz, flexible y económico; se procedió a aplicar el método analítico, dado a que, a través de su carácter cognoscitivo, permite generar nuevos criterios y teorías que contribuyen a dar una mejor solución al problema planteado.
El proceso que se llevó a cabo para su desarrollo, consta de las siguientes etapas expuestas a continuación.

\section{Recopilación de la información}

Esta primera etapa consistió en el estudio de manera general de los sistemas de monitorio y control de líquidos que se encontraban en existencia, los cuales dependiendo al contexto y finalidad variaban con respecto a su funcionamiento y/o composición. Por ello, se hizo énfasis solo en las especificaciones técnicas de sus componentes, como resultado se obtuvo información relevante acerca de las distintas formas en las que se puede abordar una situación en mayor o menor medida similar. Asimismo, se estudió la posibilidad de utilizar otro tipo de componentes a los previamente estudiados, con el motivo de que estos propiciaran una mayor optimización en los recursos del sistema, eficiencia, eficacia, flexibilidad, y que fuesen de fácil adquisición.

\section{Análisis de la estructura y funcionamiento del sistema con base en el estudio de sus partes}

La finalidad de esta siguiente etapa fue observar las distintas formas de estructurar un sistema de este tipo. Para ello, con base en los componentes examinados en la etapa anterior, se llevó a cabo un contraste entre cada uno de estos, analizando el posible comportamiento que tendrían al ser aplicados en el sistema, y como sus características intrínsecas afectarían el funcionamiento, tales como su dimensión, voltaje de operación, interfaz, corriente de alimentación, el uso de complementos, entre otros. A partir de este análisis se determinó que la creación del sistema puede llevarse a cabo con la incorporación y selección de diferentes componentes, por lo cual su desarrollo e implementación puede variar notoriamente, requiriendo en algunos casos mayor control en el aspecto físico que en el lógico, o viceversa.

\section{Análisis del problema}

Para continuar con la siguiente etapa, enfocada a dar alternativas de solución al problema planteado, antes fue necesario comprender ampliamente el contexto en el que se abordaba, verificando de esta forma su existencia, al aplicar distintas técnicas de análisis que permitieron examinar a profundidad sus factores más importantes:

BÁEZ-IBARRA, Alejandro, ENRÍQUEZ-NICOLÁS, Mario, LUNASALINAS, Francisco Antonio y ROJAS-SANTOS, Guadalupe. Sistema de Monitoreo y Control Automatizado de Nivel para Contenedores de Líquidos no Corrosivos, de Uso Doméstico y Comercial. Revista del Desarrollo Tecnológico. 2019 
1. Los efectos que ocasiona.

2. Las causas que lo provocan.

Una vez definido estos dos aspectos, se hizo posible desglosar en proporciones más simples el problema principal, lo cual hizo más rápido y preciso el proceso de identificación y selección de posibles soluciones.

\section{Identificación de alternativas de solución}

Tras el análisis de los factores involucrados en el problema planteado, se tuvieron las pautas para la formulación de las posibles propuestas de solución; para ello se aplicaron una serie de filtros sobre las alternativas seleccionadas, descartando rigurosamente cada una de ellas, hasta optar por la que más se adecuaba al contexto del problema.

En otras palabras, después de llevar a cabo un gran número de revisiones, se seleccionó aquella propuesta que brindará, la fiabilidad y eficiencia necesaria para implementar el sistema, basándose principalmente en los siguientes criterios: dimensión, resistencia, corriente de alimentación, y costo, esto con respecto a los componentes electrónicos que se utilizarían, del mismo modo se consideró como un criterio fundamental el proceso de selección de las plataformas o entornos de desarrollo que más se adecuaban a la situación.

\section{Herramientas utilizadas para la implementación de la solución del problema}

Para llevar a cabo la implementación de la propuesta de solución previamente seleccionada, se hizo uso de las siguientes herramientas:

1. Componentes electrónicos, entre los principales se encuentran:

$\begin{array}{ll}- & \text { Arduino Uno } \\ - & \text { Módulo Ethernet ENC28J60 } \\ -\quad & \begin{array}{l}\text { Sensor ultrasónico resistente al agua } \\ \text { JSN-SR04T }\end{array}\end{array}$

- Relevador

- $\quad$ Cable Ethernet UTP 8 Cat 5
2. Plataformas o Entornos de desarrollo Integrado (IDEs, por sus siglas en inglés).

- $\quad$ Arduino Software (IDE). Esta plataforma electrónica se encuentra enfocada en la programación de un microcontrolador, por lo cual integra un conjunto de herramientas de programación para tal fin.

- $\quad$ LabVIEW. Esta plataforma consiste en el diseño de sistemas hardware y software, es decir, permite la conexión, sincronización, y ejecución de un circuito completo, así como de su interfaz gráfica.

3. Complementos.

UIPEthernet. Biblioteca de Ethernet para ENC28J60.

- Ultrasonic. Biblioteca para el módulo de ultrasonido de Arduino.

\section{Diseño del Sistema de Monitoreo y Control Automatizado de Nivel para Contenedores de Líquidos no Corrosivos, de Uso Doméstico y Comercial (Circuito)}

En función a las características propias de los componentes electrónicos, el diseño del sistema a nivel físico, se realizó a través de la integración sucesiva de cada una de sus partes, verificando su correcta funcionalidad al momento de agregar un nuevo componente.

Se comenzó por llevar a cabo la conexión entre la placa de Arduino UNO con el módulo Ethernet ENC28J60, esto con el motivo de establecer desde un principio comunicación en red entre la parte física del sistema con su interfaz gráfica o panel de monitoreo y control, prescindiendo así de la necesidad de realizar una conexión directa y cableada entre ambos, por lo que su interacción sería efectuada remotamente dentro de una misma red. Después de corroborar la funcionalidad de las primeras conexiones y seleccionar apropiadamente las bibliotecas compatibles para mejorar el uso del Sensor ultrasónico resistente al agua JSN-SR04T; éste se incorporó a la estructura del sistema, conectándose directamente al microcontrolador, es decir, a la placa de Arduino UNO. 
En general, todas las conexiones entre componentes fueron realizadas siguiendo el mismo proceso. Cabe destacar que, los distintos protocolos y bibliotecas utilizados para establecer la comunicación entre cada componente, fueron relevantes para el diseño final, por lo que para probar su funcionamiento se tuvo que programar parte del código que les indicaba la funcionalidad requerida para el sistema en sí.

\section{Diseño del Sistema de Monitoreo y Control Automatizado de Nivel para Contenedores de Líquidos no Corrosivos, de Uso Doméstico y Comercial (Interfaz gráfica)}

Una vez terminado el diseño del sistema en el nivel físico, y programado parte de las acciones a realizar para cada uno de los componentes, mediante el uso del Arduino Software (IDE); se diseñó la interfaz gráfica del sistema a través del uso de la plataforma LabVIEW, la misma que mostraría y controlaría de manera remota ( sin la necesidad de que esta, permaneciese junto al circuito) la cantidad de líquido contenido en un tanque; en este caso con una capacidad de 1,100 litros, y una altura de $1.50 \mathrm{~m}$, esto mediante los valores obtenidos por el sensor ultrasónico, que reflejarían dicha cantidad.

Asimismo, dependiendo al estado del tanque, si este se encuentra vacío o lleno; el relevador contenido en el circuito actúa como un interruptor, prendiéndose y apagándose, según sea el caso, es decir, permitiendo o denegando el paso del líquido al contenedor. Es importante resaltar que, durante el diseño y programación de la interfaz gráfica, se hizo imprescindible el uso de determinadas variables estáticas, por ejemplo, al definir la dirección IP del módulo Ethernet y el número de puerto que usaría o al establecer valores por defecto cuando el sistema es ejecutado por primera vez, entre otros casos.

\section{Prototipado}

Para la elaboración y diseño del prototipo se tuvo que llevar a cabo la soldadura entre los distintos componentes, de tal forma que su tamaño final una vez unificados fuese lo más compacto posible, aumentado en gran medida el espacio libre dentro de su contenedor que los mantendría en resguardo de factores externos, entre algunos como la intemperie, altas temperaturas, fuertes viento, lluvias ácidas.
Posterior al ensamblado de los componentes, se perforó el contenedor para permitir la salida del cabezal del sensor ultrasónico al exterior, y la salida del cable ethernet que estaría conectado al modem, router, o switch más cercano, en este caso se utilizó un router; con esto se consiguió aumentar la protección, y disminuir la corrosión en los componentes por causas externas.

\section{Resultados}

El proceso que se realizó para la creación del sistema, consistió en conocer en primer lugar, cuáles eran las opciones de componentes más óptimos para desarrollar un sistema con las siguientes características:

$\begin{array}{ll}\text { - } & \text { Visualización y control mediante interfaz } \\ \text { gráfica (Panel de Monitoreo y Control). } \\ \text { - } \quad \text { Comunicación y acceso a datos a } \\ \text { distancias largas. } \\ \text { - } \quad \text { Eficiencia en su funcionamiento. } \\ -\quad \text { Seguridad en sus mediciones. } \\ -\quad \text { Resistencias a factores externos. } \\ -\quad \text { Implementación en diferentes entornos: } \\ \text { doméstico y comercial. } \\ \text { Tamaño reducido o compacto } \\ \text { (ensamblaje a una sola pieza). } \\ \text { Bajo costo. }\end{array}$

De modo que, el sistema fue desarrollado inicialmente con la implementación del módulo Ethernet ENC28J60, cuya comunicación es mediante el protocolo denominado Interfaz Periférica Serial (ISP, por sus siglas en ingles), este protocolo es utilizado para comunicar un microcontrolador con otro y con periféricos.

En este tipo de comunicación siempre se encuentra presente un dispositivo llamado maestro, por lo general suele ser un microcontrolador, y uno o más de un periférico que es controlado por este.

Aquí fue necesaria la realización de una adecuada conexión con Arduino Uno, debido a que dependiendo a la placa a utilizar su conexión ya se encuentra determinada por defecto, en este caso con los pines 10 (CS), 11 (SI), 12 (SO), y 13 (SCK), como se muestra en la siguiente figura. 


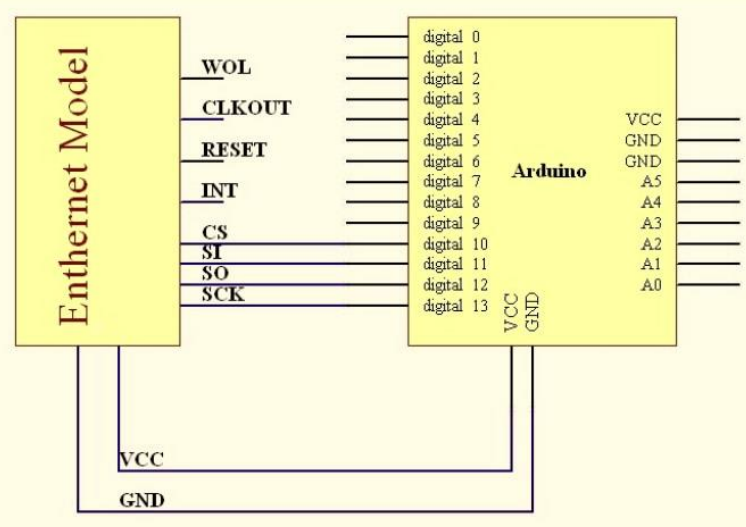

Figura 1 Conexión

Fuente: http://tecnotinker.blogspot.com/2012/07/uso-delmodulo-ethernet-enc28j60-con.html

\section{El módulo Ethernet ENC28J60 a} diferencia del Ethernet shield Wiznet W5100 con un costo más elevado no es compatible con la biblioteca Ethernet propia de Arduino, no obstante, tiene completa compatibilidad con la biblioteca UIPEthernet, la cual se utilizó para establecer interacción entre todos los componentes que constituyen la parte física del sistema, y el panel. Esta característica se logró a partir de establecer comunicación en red utilizando los protocolos TCP/IP, y como resultado se obtuvo un sistema con una mayor flexibilidad para ser aplicado en distintos entornos al contar con la capacidad de ser monitoreado y controlado remotamente dentro de una misma red.

Con base en lo anterior, las acciones para establecer dicha comunicación fueron: la asignación de la dirección IP 10.185.127.149, la dirección MAC 0x1A, 0xAE, 0xBE, 0xEF, 0xEF, 0xDE, y el puerto número 8000 como valores estáticos a la parte física del sistema formada por los componentes electrónicos en cuestión; y la verificación de que estos datos fueron cargados correctamente, para lo cual se efectuaron pruebas de respuesta, como la ejecución del comando ping en la consola de Windows, corroborando que la dirección IP especificada era accesible desde red.

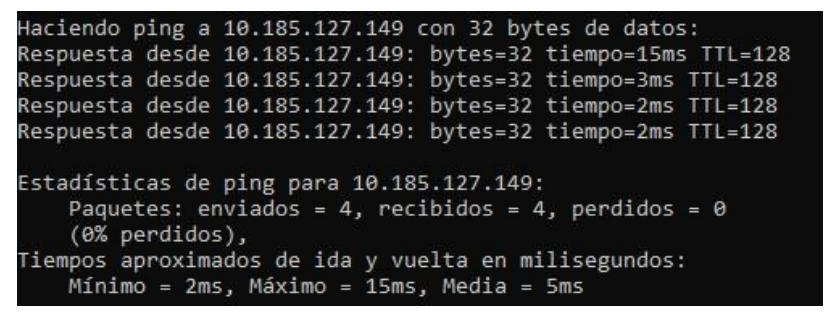

Figura 2 Verificación de la accesibilidad de la dirección IP 10.185.127.149 en red

Fuente: Elaboración Propia
Las instrucciones para poder establecer los datos de configuración del acceso en red, fueron programados desde el IDE de Arduino, en donde además de inicializar los valores, antes fueron agregadas las bibliotecas UIPEthernet $\mathrm{y}$ Ultrasonic.

La primera permitió la creación de tres tipos de objetos IPAddress, EthernetServer, y EthernetClient, este último encargado específicamente de recibir y enviar información obtenida por el sensor ultrasónico al panel; la segunda por su parte repercutió en la mejora del tiempo de ejecución entre los procesos del sistema (lecturas, entradas, salidas, y cálculos) al interactuar con el sensor ultrasónico, y proporcionar lecturas más rápidas y con una mejor precisión.

La conexión del sensor ultrasónico era determinada por los pines: Echo (TX) y Trigger (RX), los cuales fueron conectados a los pines digitales 7 y 6 de la placa de Arduino UNO, respectivamente, dado a que el número de pin no alteraba el funcionamiento del sensor, como en el caso del módulo de Ethernet, era indiferente el pin que se fuese a utilizar.

Otro aspecto importante en las lecturas del sensor ultrasónico, fue que presenta un rango de detección de 25 a $450 \mathrm{~cm}$ aproximadamente, lo que se solucionó a través de la plataforma LabVIEW al realizar los cálculos y validaciones considerando este límite.

Al estar usando dos entornos para la codificación de los procesos del sistema, el Arduino Software (IDE) y LabVIEW, en ocasiones era posible seleccionar en cuál de los dos se realizaba una determinada instrucción, debido a esto se consideraron aspectos de rendimiento, ejecución y de aplicación.

De igual modo, se presentó la necesidad de que ciertos valores fuesen utilizados y manipulados en ambos entornos, tal es el caso del encendido y apagado del relevador conectado al Arduino por medio del pin digital 9 , por lo que, para iniciar, detener o continuar con las lecturas de los valores del sensor ultrasónico, se definió una condicionante a través de un valor que ambos entornos compartían. 
Archivo Éditar Programa Herramientas Ayuda

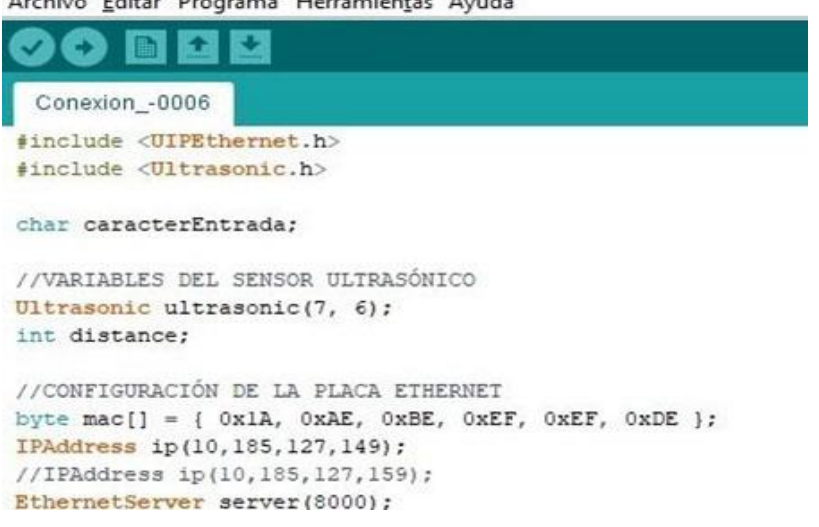

Figura 3 Código parte 1

Fuente: Elaboración Propia

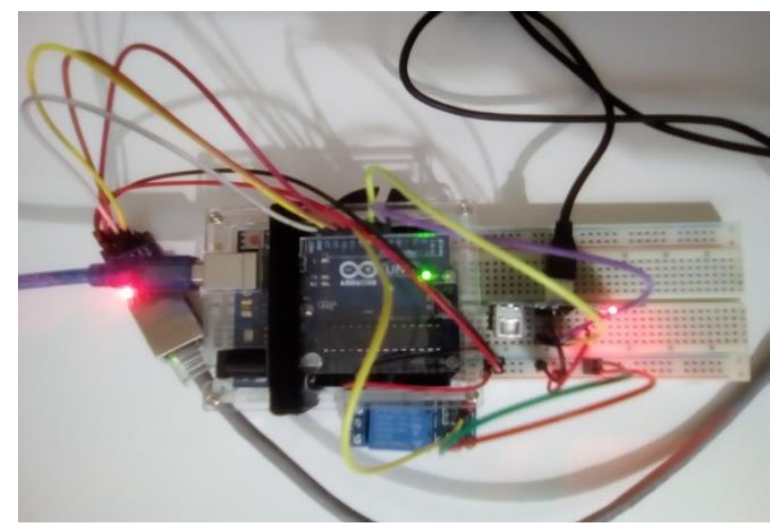

Figura 4 Conexión Arduino Fuente: Elaboración Propia

El tipo de programación que se empleó para la realización de la interfaz gráfica de monitoreo y control fue mediante bloques, LabVIEW permite una codificación gráfica, con la posibilidad de agregar código en los bloques si así se requiere; en este caso, la programación fue en su mayoría gráfica, pero también se programó un fragmento de código dentro de un bloque.

LabVIEW posee sus propios controles y elementos para el diseño e interacción entre los distintos componentes electrónicos, por ejemplo, los más utilizados son numeric, boolean, string, y sus variaciones solo por mencionar algunos; facilitando en cierta medida la creación de interfaces graficas. Para el sistema en específico se utilizaron los siguientes controles y elementos: Flat Sequence Structure, Case Structure, While Loop, Tab Control, Opcion, Formula Node, Slide, entre otros; tanto para ingresar datos como para mostrarlos.

Por consiguiente, es recomendable tener un conocimiento previo acerca del uso de esta herramienta para poder utilizarla con mayor facilidad y crear interfaces mucho más robustas.
Para la comunicación en red, LabVIEW posee componentes específicos con el protocolo $\mathrm{TCP} / \mathrm{IP}$, en donde es necesario abrir y cerrar la comunicación cada vez que se cancele la ejecución del sistema, además de definir la dirección IP y el número de puerto. Una característica flexible del sistema es que estos valores se definieron en su inicio como valores inmutables, pero que posteriormente cambiaron a ser valores que el usuario podría configurar, al igual que la altura del tanque, variable enfocada a definir el rango de distancia que tiene el sensor ultrasónico con respecto al contenedor.

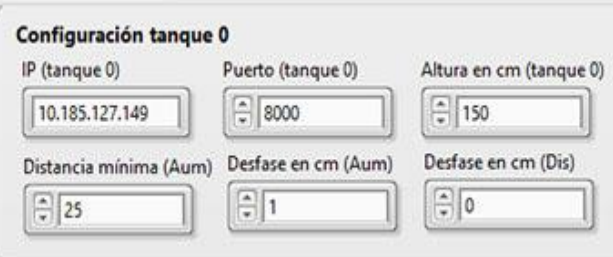

Figura 5 Configuración de la placa Ethernet ENC28J60 y control

Fuente: Elaboración Propia

Durante la etapa de desarrollo de la interfaz gráfica del sistema se fueron realizando varias verificaciones de uso, a través de las cuales se examinó y observó el comportamiento completo de todas las partes, con lo cual se optimizaron los métodos hasta el punto de mejorar la sincronización entre las lecturas, y operaciones de los procesos; aunado a ello, se agregó más información sobre el estado del contenedor en el panel del sistema, creando a su vez una señalización del nivel de líquido por medio del color del tanque (0-40\% azul, 41-80\% naranja, $80-100 \%$ verde, en porcentajes de llenado), y del estado del relevador (0-99\% apagado, 100\% encendido), e información extra sobre la distancia vacía total.

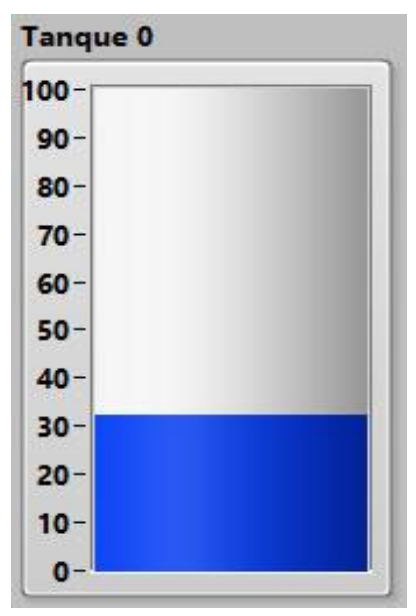

Figura 6 Porcentajes de llenado (0-40\% azul) Fuente: Elaboración Propia

BÁEZ-IBARRA, Alejandro, ENRÍQUEZ-NICOLÁS, Mario, LUNASALINAS, Francisco Antonio y ROJAS-SANTOS, Guadalupe. Sistema de Monitoreo y Control Automatizado de Nivel para Contenedores de Líquidos no Corrosivos, de Uso Doméstico y Comercial. Revista del Desarrollo Tecnológico. 2019 


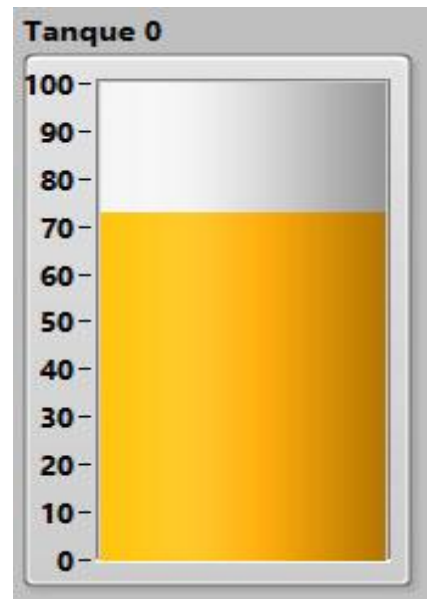

Figura 7 Porcentajes de llenado (41-80\% naranja) Fuente: Elaboración Propia

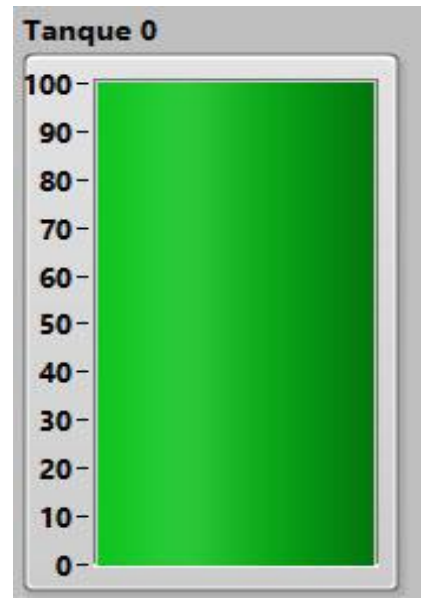

Figura 8 Porcentajes de llenado (80-100\% verde) Fuente: Elaboración Propia

En la siguiente figura se puede observar la interfaz final, el panel de control, el sistema en funcionamiento, y el diagrama en bloques.

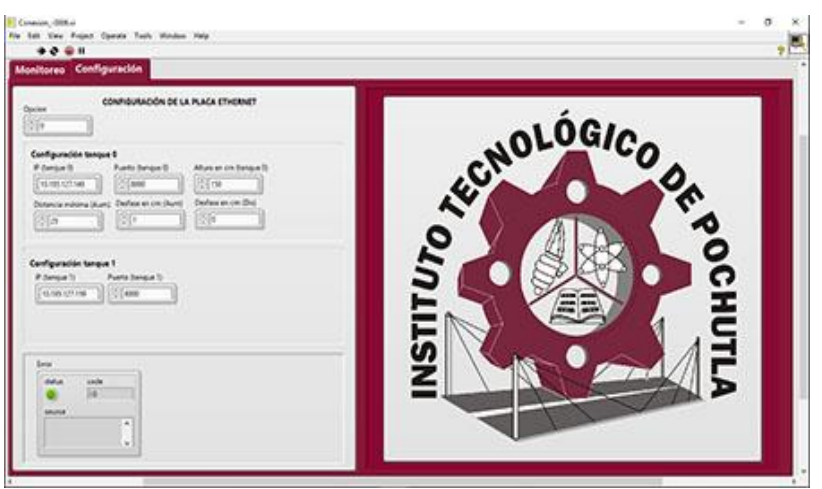

Figura 7 Panel de monitoreo y control Fuente: Elaboración Propia

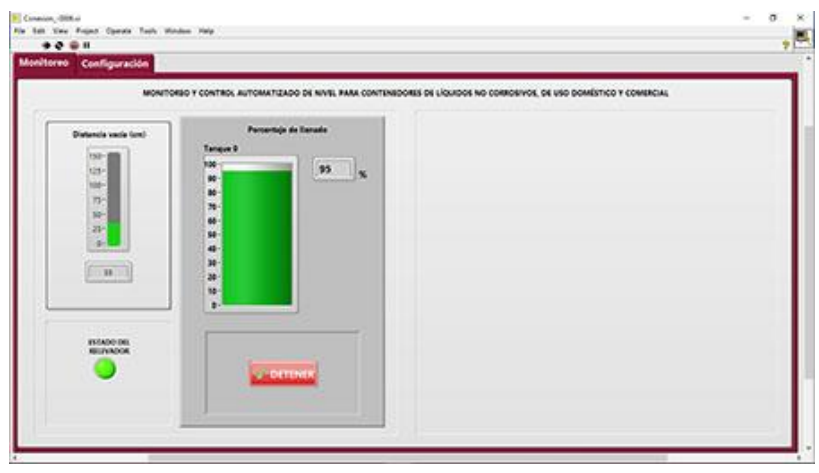

Figura 8 Sistema de monitoreo y control automatizado Fuente: Elaboración Propia

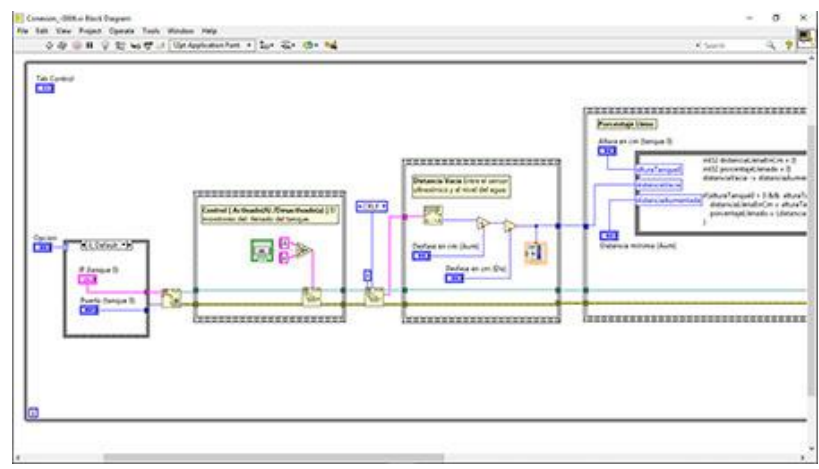

Figura 9 Diagrama en bloques del Sistema de monitoreo y control automatizado

Fuente: Elaboración Propia

\section{Conclusiones}

Con base en los resultados obtenidos, se hace posible afirmar que la creación de este sistema automatiza en gran medida una de los procesos que requiere mayor atención por parte de las personas, como lo es el monitoreo y control del llenado de contenedores de líquidos, y lo que hace que este sistema sea factible de usar, es que fue creado considerando aspectos generales que pueden ser aplicados en diferentes entornos, $\mathrm{y}$ con un precio asequible.

Una de las características que presenta, es su escalabilidad, debido a que es posible la implementación de nuevos controles, nuevas formas de medición, nuevos sensores, que se pueden agregar según las especificaciones de cada usuario, estos podrían implementarse de forma genérica o bien personalizada.

\section{Agradecimiento}

Un especial agradecimiento a nuestras instituciones: I. T. Pochutla y al I.T Salina Cruz, por la facilidad que nos brindaron para el desarrollo de este nuevo sistema. 


\section{Referencias}

Fernando, J. C. (2005). CONEXIÓN TCP/IP ENTRE DOS ESTACIONES USANDO LABVIEW 7 EXPRESS. [En línea] Disponible en: http://webcervices.weebly.com/uploads/1/5/ 0/3/15034326/conexi-n20tcp_ip20entre20dos20 estaciones20usando20labview20720express.pdf [Consultado el 7 de junio del 2019]

LabVIEW MakerHub. (s.f). LINX VI Reference. [En línea] Disponible en: https://www.labviewmakerhub.com/doku.php?i $\mathrm{d}=$ learn:libraries:linx:reference:labview:start [Consultado el 12 de junio del 2019]

LabVIEW.pdf [Consultado el 12 de junio del 2019]

Llamas, Luis. (2016). MANEJAR CARGAS DE MÁS DE 220V CON ARDUINO Y SALIDA POR RELÉ. [En línea] Disponible en https://www.luisllamas.es/arduino-salida-rele/ [Consultado el 7 de junio del 2019]

Murcia. G. (s.f). Comunicación Ethernet con Arduino y LabVIEW. [En línea] Disponible en: http://www3.fi.mdp.edu.ar/electrica/instrument acion/recursos/ia2016_clase13_guia13.pdf [Consultado el 12 de junio del 2019]

National Instruments. (2018). Basic TCP/IP Communication in LabVIEW. [En línea] Disponible en: http://www.ni.com/whitepaper/2710/en/ [Consultado el 7 de junio del 2019]

Naylamp, Mechatronics SAC, (s.f). Tutorial Módulo Ethernet ENC28J60 y Arduino. [En línea] Disponible en: https://naylampmechatronics.com/blog/17_Tuto rial-M\%C3\%B3dulo-Ethernet-ENC28J60-y-

Arduino.html [Consultado el 7 de junio del 2019]

Ruiz, G. J. M. (s.f). Utilización de Labview para la Visualización y Control de la Plataforma Open Hardware Arduino. [En línea] Disponible en: $\quad$ http://proyecto987.es/blog/wpcontent/uploads/2016/04/Arduino-

Tecnotinker. (2012). Uso del módulo ethernet ENC28J60 con Arduino: Parte 1. [En línea] Disponible en: http://tecnotinker.blogspot. com/2012/07/uso-del-modulo-ethernet-

enc28j60-con.html [Consultado el 7 de junio del 2019].
Valle, H. L. (s.f). Sensor de nivel de agua con Arduino. [En línea] Disponible en: https://programarfacil.com/blog/arduinoblog/sensor-de-nivel-de-agua-con-arduino/ [Consultado el 12 de junio del 2019]
BÁEZ-IBARRA, Alejandro, ENRÍQUEZ-NICOLÁS, Mario, LUNASALINAS, Francisco Antonio y ROJAS-SANTOS, Guadalupe. Sistema de Monitoreo y Control Automatizado de Nivel para Contenedores de Líquidos no Corrosivos, de Uso Doméstico y Comercial. Revista del Desarrollo Tecnológico. 2019 